\title{
ATRIBUIÇÕES DE IDENTIDADE: O TERCEIRO MUNDO, VISTO DO PRIMEIRO, SEGUNDO FREDRIC JAMESON
}

\author{
José Luis Jobim*
}

\begin{abstract}
RESUMO: Analisando o ensaio Third word Literature in the Era of Multinational Capital, de Fredric Jameson, buscaremos depreender como se configuram os conceitos a partir dos quais se formularão os julgamentos sobre o que o autor norte-americano identifica como "literatura do terceiro mundo".
\end{abstract}

PALAVRAS-CHAVE: Identidade da literatura; terceiro mundo.

Wo contexto atual, não é fácil para a teoria e a crítica

literárias trabalharem conceitualmente com os fundamentos dos quadros de referência a partir dos quais se formulam os próprios julgamentos dos críticos e teóricos. Nas linhas que se seguem, buscaremos colocar em questão as premissas a partir das quais Fredric Jameson alega estar descrevendo "a literatura do terceiro-mundo na era do capitalismo multinacional”.

Escolhemos, como objeto de nossa atenção, o seu ensaio "Third word Literature in the Era of Multinational Capital", publicado na revista Social Text, em 1986. Como se verá, a análise deste ensaio

Universidade do Estado do Rio de Janeiro - UERJ e Universidade Federal Fluminense - UFF. 
nos permitirá inclusive desenvolver muitos aspectos associados a nosso tema principal.

Começaremos nosso percurso crítico sobre o ensaio de Jameson, observando que a própria escolha do termo third word já é problemática, pois este começou a ser empregado nos anos 50, na França, por analogia com o terceiro estado (que no sistema politico anterior à Revolução Francesa englobava todos os que não pertenciam à nobreza ou ao alto clero, ou seja, englobava a maioria da população francesa da época). O termo ganhou circulação internacional em contextos tanto acadêmicos quanto politicos, referindose tanto aos movimentos nacionalistas anticoloniais dos anos 50 até os 70 quanto à análise político-econômica da teoria da dependência e da teoria do sistema do mundo (André Gunder Frank, Immanuel Wallerstein, Samir Amin). (Shoat, 1992: 100)

Sendo o termo problemático, torna-se relevante destacar a justificativa produzida por Jameson para seu uso:

A final observation on my use of the term "third word." I take the point of criticisms of this expression, particularly those which stress the way in which it obliterates profound differences between a whole range of non-western countries and situations [...]. I don't however, see any comparable expression that articulates, as this one does, the fundamental breaks between the capitalist first world, the socialist bloc of the second world, and a range of other countries which have suffered the experience of colonialism and imperialism. [...] I am using the term "third world" in an essentially descriptive sense, and objections to it do not strike me as especially relevant to the argument I am making. (Jameson, 1986: 67)

Observe-se que Jameson, ao afirmar que está usando o termo terceiro mundo "em um sentido essencialmente descritivo", acaba ignorando que a descrição não é nunca ideológica ou cognitivamente neutra, como apontou Aijaz Ahmad, em sua crítica ao texto 
de Jameson, pois descrever é especificar um lugar de sentido, construir um objeto de conhecimento e produzir um conhecimento que será limitado pelo ato de construção descritiva. Ahmad lembra que a "descrição" teve um papel importante no discurso colonial, já que foi pela acumulação de descrições - dos corpos, dos atos de fala, dos habitats, dos conflitos e desejos, das políticas, das sexualidades dos colonizados -, em campos como a Etnologia, a Ficção, a Fotografia, a Lingüística, a Ciência Política, que o discurso colonial foi capaz de classificar e dominar ideologicamente o sujeito colonial, capacitando-se a transformar a multiplicidade e diferença, descritivamente verificável, em hierarquia de valor ideologicamente percebida:

Dizer, em resumo, que o que se apresenta é "essencialmente descritivo" é afirmar um nivel de facticidade que escamoteia sua própria ideologia, e preparar um terreno a partir do qual julgamentos de classificação, generalização e valor podem ser feitos. (Ahmad, 1987: 6)

Com efeito, Jameson cria uma imagem de literatura do terceiro mundo como sendo, ao mesmo tempo, derivada, inferior esteticamente e atrasada em relação à do primeiro mundo, embora alegue que sua intervenção, com a publicação do artigo, foi "progressista”, visando, entre outras coisas, a chamar a atenção sobre "a necessidade de ensinar as literaturas do terceiro mundo". ${ }^{1}$ Mas,

\footnotetext{
Em sua "Brief Response" ao artigo de Aijaz Ahmad, Jameson declara: “...I still think my intervention was a positive and progressive one, whose implications (on any number of levels) include: the necessity for teaching third word literatures; the recognition of the challenge they pose to even the most advanced contemporary theory; the need for a relational way of thinking global culture (such that we cannot henceforth think 'first-world' literature in isolation from that of other global spaces); the proposal for a comparative study of cultural situations [...]; and finally, the suggestion [...] that when we get done with all that we may want to entertain the possibility that we also need a (new) theory of second world culture as well." (Jameson, 1987, p. 27)
} 
para que possamos fundamentar este nosso juizo, recordemos as palavras do ensaista norte-americano:

The third word novel will not offer the satisfaction of Proust or Joyce; what is more damaging than that, perhaps, is its tendency to remind us of outmoded stages of our own firstworld cultural development and to cause us to conclude that "they are still writing novels like Dreiser or Sherwood Anderson." (Jameson, 1986: 65)

Indeed our want of sympathy for these often unmodern third word texts is itself frequently but a disguise for some deeper fear of the affluent about the way people actually live in other parts of the world - a way of life that still has little in common with daily life in the American suburb. There is nothing particularly disgraceful in having lived a sheltered life, in never having had to confront the difficulties, the complications and the frustrations of urban living, but it is nothing to be particularly proud of either. Moreover, a limited experience of life normally does not make for a wide range of sympathies with very different kinds of people (I'm thinking of differences that range from gender and race all the way to those of social class and culture).

The way in which this affects the reading process seems to be as follows: as western readers whose tastes (and much else) have been formed by our own modernisms, a popular or socially realistic third word novel tends to come before us, not immediately, but as though already-read. We sense, between ourselves and this alien text, the presence of another reader, of the Other reader, for whom a narrative, which strikes us as conventional or naïve, has a freshnes of information and a social interest that we cannot share. The fear and the resistance I'm evoking has to do, then, with the sense of our own noncoincidence with that Other reader, so different from ourselves; our sense that to coincide in any adequate way with that Other "ideal reader" - that is to say, to read this text adequately - we would have to give up a great deal that is individually precious 
to us and acknowledge an existence and a situation unfamiliar and therefore frightening - one that we do not know and prefer not to know. (Jameson, 1986: 66)

Chamamos a atenção, inicialmente, para o uso do us - com seu efeito discursivo de inclusão virtual do seu público leitor, o qual, segundo o próprio Jameson, seria um público de "primeiro mundo", basicamente norte-americano ${ }^{2}$. Depois, a referência explicita aos modelos (Proust e Joyce) em relação aos quais a produção literária terceiro-mundista pareceria com um estágio fora de moda de nosso próprio [deles, primeiro-mundistas] desenvolvimento cultural primeiro-mundista. Interessante observar como a articulação de estágio, desenvolvimento e primeiro mundo na citação assemelha-se profundamente a tantas outras articulações, que basicamente pagam tributo à visão de que existem "estágios" para o "desenvolvimento", em relação aos quais determinados estados-nação encontram-se sincronizados (os paises "desenvolvidos", do "primeiro mundo") e outros não (os países "subdesenvolvidos" ou "em desenvolvimento", do "terceiro mundo"). Com freqüência se utiliza esta visão para argumentar que há um determinado caminho para o "desenvolvimento" que passa necessariamente por certas etapas ("estágios") - modelizadas à feição da história do "primeiro mundo" - cuja ultrapassagem sucessiva poderia conduzir ao modelo desejável de "atualização", de aggiornamento. (No Brasil, podemos observar como a luta pela implantação desta visão tem sido articulada discursivamente em torno de uma argumentação que alega ser necessário queimarmos etapas para nos modernizarmos). ${ }^{3}$

2 Cf. JAMESON, 1987, p. 26: "The essay was intended as an intervention into a 'first-world' literary and critical situation, in which it seemed inportant to me to stress the loss of certain literary functions and intellectual commitments in the contemporary American scene".

3 Cf. BENJAMIN et al, p. 69: "Avaliamos nosso modelo econômico pelo grau em que ele consegue copiar aspectos do modelo dos paises mais ricos, sem nos perguntarmos se esse processo conduzirá à superação de nossas próprias carências e ao melhor aproveitamento de nosas potencialidades. Aceleramos uma integração internacional subordinada, construida às custas da desintegração nacional". 
JOBIN, José Luís. Atribuições de identidade: o terceiro mundo, visto do primeiro, segundo...

E já que falamos em modelização, é importante assinalar também que o argumento de Jameson - acusando a literatura do terceiro mundo de mimetizar um realismo que já estaria ultrapassado no primeiro mundo - apresenta problemas dos dois lados. Não só é incorreto apontar esta característica para toda a literatura "terceiro-mundista", como também não seria exato apontar o descarte do realismo pela literatura do "primeiro mundo":

Some of the most highly regarded US fictionists of the present cultural moment, from Bellow and Malamud to Grace Paley and Robert Stone, seem to write not quite "like Dreiser or Sherwood Anderson" but surely within the realist mode. On the other hand, Cesaire became so popular among the French surrealists because the terms of his discourse were contemporaneous with their own, and Neruda has been translated by some of the leading poets of the US because he is even formally not "outmoded." Novelists like Marquez or Rushdie have been so well received in the US/British literary circles precisely because they do not write like Dreiser or Sherwood Anderson; the satisfaction of their outrageous texts are not those of Proust or Joyce but are surely of an analogous kind, delightful to readers brought up on modernism and postmodernism. Cesaire's Return to the Native Land is what it is because it combines what Jameson calls a "national allegory" with the formal methods of the Parisian avant-garde of his students days. Borges is of course not seen in the US any longer in terms of his latin american origin; he now belongs to the august company of the significant moderns, much like Kafka. (Ahmad, 1987: 17)

É relevante também a estratégia discursiva de Jameson, construída na base de especulações sobre a recepção da literatura terceiro-mundista por um público virtual, no qual ele também se inclui - pelo uso da primeira pessoa do plural -, e ao qual faz atribuições, isto é, atribui um medo profundo, que os ricos teriam, do modo "diferente" como vivem os de "outras partes do mundo". Dis- 
to resultaria a falta de identificação ou simpatia com o outro, com sua cultura, com seu modo de ser, com sua diferença enfim. Portanto, pode-se presumir que Jameson advoga a presença de textos do "terceiro mundo" nos Estados Unidos, para que, por meio deles, se incorpore a experiência do/com o outro, permitindo o exercício da simpatia e do respeito às diferenças culturais.

Contudo, é curioso observar sua narrativa sobre o modo como ele supõe desencadear-se o processo de leitura dos romances de terceiro mundo. Para começar, ele acha que o gosto dos leitores "ocidentais" [suponho que do "primeiro mundo"] foi formado pelos modernismos, o que faria um romance realista do terceiro mundo ser percebido como coisa já vista. Obviamente, tanto a primeira parte de sua argumentação é discutivel - será mesmo que o gosto dos leitores "ocidentais" é modernista? - quanto a segunda merece reparos, pois também tem implicações derivadas do questionamento da primeira. ${ }^{4}$

4 De algum modo, esta formulação de Jameson, em termos de realismo versus modernismo parece ecoar a análise que este ensaista fez, em 1977, do debate Brecht-Lukács: "Within the Marxism of this century, the precipitant of the controversy over realism and modernism was the living fact and persisting influence of expressionism among the writers of the German Left in the 1920s and 30s. An implacable ideological denunciation by Lukács in 1934 set the stage for the series of interconnected debates and exchanges between Bloch, Lukács, Brecht, Benjamin, and Adorno generally referred as the "realism debate." Much of the fascination of these jousts, indeed, comes from the internal dynamism by which all the logical possibilities are rapidly generated in turn, so that it quickly extends beyond the local phenomenon of expressionism, and even beyond the ideal type of realism itself, to draw within its scope the problems of popular art. naturalism, socialist realism, avant-gardism, media, and finally modernism-political and nonpolitical - in general. Today, many of its fundamental themes and concerns have been transmited by the Frankfurt School, and in particular by Marcuse, to the student and antiwar movements of the 60s, while the revival of Brecht has ensured their propagation among political modernisms of the kind exemplified by the Tel Quel group." (Jameson, 1988 [1977], p. 133-4)

É interessante assinalar, naquele artigo de 1977, que Jameson também ressalta dois aspectos da obra de Jean-Paul Sartre, O que é a literatura (1948), que o próprio critico norte-americano acabará adotando: a noção de engajamento (engagement [fr.], commitment [ingl.]), e a exclusão da poesia e da lírica (é notável o foco de sua argumentação em narrativas). 
JOBIN, José Luís. Atribuições de identidade: o terceiro mundo, visto do primeiro, segundo...

Não bastasse tudo isto, ainda há a construção da imagem do outro leitor terceiro-mundista, "para quem a narrativa, que nos parece convencional ou ingênua" [a "nós" primeiro-mundistas?], tem a frescura da novidade e um interesse social que não podem ser compartilhados pelos leitores do primeiro mundo. Para começar, será possivel fazer generalizações desta ordem sobre os leitores do "primeiro mundo"? Talvez o modelo textual com o qual este leitor esteja mais acostumado seja ainda o realista ou semelhante, pois é mais ou menos de acordo com as convenções deste estilo que são construídos os discursos não só informativos cotidianos (dos jornais, revistas etc.), mas também aqueles com pretensões descritivas (da História, Economia, Sociologia, Antropologia etc.).

Acrescente-se também que Jameson, dez anos antes (Jameson, 1988 [1977]), expressa a opinião de que a originalidade do conceito de realismo residiria em sua aspiração a ter um status cognitivo, além de estético. O ideal de realismo - um novo valor, contemporâneo à secularização do mundo sob o capitalismo - pressuporia uma forma de experiência estética que ainda alegaria uma relação estreita com o próprio real, isto é, com aqueles domínios do conhecimento e da praxis que tinham tradicionalmente sido diferenciados do dominio do estético, com seus julgamentos desinteressados e sua constituição como pura aparência. Contudo, segundo o ensaísta norte-americano, seria extremamente dificil fazer justiça àquelas duas propriedades do realismo, simultaneamente. Na prática, uma ênfase excessiva em sua função cognitiva freqüentemente conduziria a uma negação ingênua do necessário caráter ficcional do discurso artístico, ou mesmo a exortações iconoclásticas a favor do "fim da arte" em nome de uma militância política. (Jameson, 1988 [1977], p. 135)

Levantando os argumentos contrários à atitude hostil de Georg Lukács em relação ao Modernismo, Jameson alega que até os que defendem aqueles argumentos necessariamente insistem na existência de um conteúdo social reprimido, mesmo naquelas obras 
modernas que não parecem explicitar isto. O Modernismo seria então não tanto um modo de evitar o conteúdo social - "de qualquer jeito uma impossibilidade para seres como nós, que são 'condenados' à história e à implacável socialidade mesmo da mais aparentemente privada de nossás experiências" -, mas; isto sim, um modo de administrá-lo e contê-lo, colocando-o fora de vista na própria forma, por meio de técnicas específicas de enquadramento e deslocamento que poderiam ser identificadas com precisão. (Jameson, 1988 [1977], p. 138)

Também é importante ressaltar o destaque dado, naquele texto de 1977, à noção brechtiana de verfremdung, porque esta noção pode ser relacionada com a ostranenie (estranhamento) dos formalistas russos e com toda a valorização que as vanguardas do inicio do século fizeram do novo (o "make it new" poundiano). O "vínculo da história literária com a literatura contemporânea viva”, de que falou Tynianov (Toledo, 1973), por exemplo, pode ter paralelo com a relação entre o Formalismo e as vanguardas artísticas russas da época, as quais também apresentavam elos entre si. Hoje, revendo aquele contexto, talvez possamos dizer que à arte sem objeto do Cubismo corresponderia a poesia só som do Futurismo; à teoria da palavra em seu aspecto sonoro, como material e tema da poesia (enfim, ao mundo poético válido por si do Futurismo) corresponderia a imanência nos estudos literários ${ }^{5}$. Em sua análise do Formalismo Russo, Bakhtin ressaltou que aquele movimento começou na época da dissolução do Simbolismo e teve relações íntimas com as vanguardas literárias de sua época: esta sua integração com o meio literário teria, na opinião daquele teórico, influenciado a concepção de literatura e os temas de pesquisa escolhidos pelos formalistas. ${ }^{6}$

5 POMORSKA, K. Formalismo e futurismo: a teoria formalista russa e seu ambiente poético. São Paulo: Perspectiva, 1972. p. 5.

6 MEDVEDEV, P. [BAKHTIN; M.M.] The formal method in literary Scholrship. Baltimore: the Johns Hopkins University Press, 1978. p. 56. 
JOBIN, José Luís. Atribuições de identidade: o terceiro mundo, visto do primeiro, segundo...

Jameson de certa forma reformata um argumento já criticado por Bakhtin naquele ensaio de 1928. Quando afirma que o romance do terceiro mundo parece já-lido, already-read, déjà-vu ao leitor do primeiro mundo, o ensaísta norte-americano parece lançar mão - em outro contexto e com outras implicações - de uma certa linha de raciocínio, segundo a qual se presume que a percepção precisa da novidade, do up-to-date, do produto mais recente. Em outras palavras, supõe-se ser necessário que o leitor vá além do already-read (ou seja, daquilo que seu gosto identifica como outmoded, fora de moda, pertencente a um procedimento literário anterior), a fim de entrar em contato com o novo, com uma obra que provoque o "estranhamento" em relação a procedimentos literários cristalizados (ou automatizados, segundo o vocabulário dos formalistas).

No entanto, Bakhtin já apontou para o problema da psicologização neste tipo de raciocinio, que na opinião dele só poderia ser aplicado "dentro das fronteiras de um organismo único, individual" (Bakhtin, 1978 [1928], p.161). O teórico russo argumentaria que a percepção depende do que se presume ser alreadyread: para uma criança sem repertório de leituras prévias, tudo seria novo. ${ }^{7}$

7 Bakhtin acha que uma das falhas da teoria formalista do desenvolvimento literário é exatamente o fato de sua pré-condição básica ser o que ele chama de lei psicofisiológica da automatização-perceptibilidade. Como poderíamos explicar a evolução literária empregando conceitos de natureza psicofisiológica, como o par automatização/ perceptibilidade, que só podem ser aplicados dentro das fronteiras de um organismo único, individual? A lei da automatização/perceptibilidade, criada pelos formalistas, poderia até explicar a simultaneidade de formas literárias novas e velhas, mas não explicaria a sua sucessão, não explicaria a evolução literária. Além disso, se o estranhamento se faz em relação aos procedimentos automatizados, isto é, em relação ao cânon estabelecido, então, conseqüentemente, para quem não conhece este cânon, como as crianças, as pré-condições de perceptibilidade das formas literárias mais recentes ou das mais antigas seriam iguais. Acrescente-se a tudo isso o fato de que, caso a automatização dependa de quem a perceba enquanto automatização, então ela não pertence à obra em si, e sim à sua recepção pelo leitor. (Bakhtin, 1978 [1928], p. 161-5) 
Além disso, a referência do crítico norte-americano ao Modernismo torna importante também verificar em que medida o próprio julgamento de Jameson está contaminado pelo critério da frescura da novidade, ou seja, até que ponto a herança das vanguardas do início do século $\mathrm{XX}$ serve de fundamento para o próprio ensaio que estamos analisando. E é bom lembrar também que, quando ele especula que para o leitor primeiro-mundista (cujo gosto supostamente teria sido formado pelo Modernismo) o romance terceiromundista tenderia a parecer convencional ou ingênuo, embora para o leitor terceiro-mundista possa parecer novidade, podemos levantar outra questão: - Que contexto de recepção se supõe para o primeiro e o terceiro mundos?

Podemos aventar a hipótese de que Jameson supõe um horizonte de recepção realista ou pré-modernista para o terceiro mundo - aparentemente anacrônico em relação ao estágio mais "moderno" do primeiro mundo, o que explicaria o dejá-vu, already-read ou qualquer outro sintagma que aponte para reiteração de formas estéticas já conhecidas ou fora de moda, que lembram estágios anteriores do desenvolvimento cultural de primeiro mundo (remind us of outmoded stages of our own first-world cultural development). Todavia, lembremo-nos de que o already-read é uma categoria que pode ter como par simétrico o never-read-before, a aspiração à novidade, à originalidade absoluta, em suma: o ideal das vanguardas. Este ideal é frontalmente oposto, por exemplo, às poéticas da imitação e da emulação, que estiveram vigentes no Ocidente até pelo menos o século XVIII. ${ }^{8}$

Com o olhar de hoje, podemos ver uma certa ironia na situação: Bakhtin acusa um conceito central para formalistas (o de automatização) de não ser...intrinsecamente literário, pelo menos nos moldes daqueles que acusam o Formalismo Russo de pregar a auto-suficiência dos estudos literários - propondo, segundo a visão destes acusadores, a autonomia e imanência da literatura. O próprio Jameson de certa forma ecoa estas acusaçōes em The Prison-House of Language; a Critical account of Structuralism and Russian Formalism. Princeton: Princeton University Press, 1972. 
Talvez fosse mais prudente afirmar que só a partir do levantamento do repertório de leitura de um determinado público se poderia construir, com um grau menor de incertezas, qual é o universo de expectativas deste público. Ou seja, somente após delimitarmos o horizonte de expectativas de um determinado público em um determinado momento histórico poderiamos aventar hipóteses um pouco mais sólidas sobre um romance realista do "terceiro mundo" poder ou não ser percebido como exemplar de um estilo fora de moda.

Para complementar nossas breves observações sobre o ensaio de Jameson, nada melhor do que comentar as suas palavras iniciais:

Judging from recent conversations among third word intellectuals, there is now an obsessive return of the national situation itself, the name of the country that returns again and again like a gong, the collective attention to "us" and what we have to do and how we do it, to what we can't do and what we do better than this or that nationality, our unique characteristics, in short to the level of the "people." This is not the way American intellectuals have been discussing "America", and indeed one might feel that the whole matter is nothing but that old thing called "nationalism", long since liquidated here and rightly so. Yet a certain nationalism is fundamental in the Third word (and also in the most vital areas of the second world), thus making it legitimate to ask whether it is all that bad in the end. Does in fact the message of some disabused and more experienced first-world wisdom (that of Europe even more that of the United States) consist in urging these nation states to outgrow it as fast as possible? The predictable reminders of Kampuchea and Iran do not really seem to me to settle anything or suggest by what these nationalisms might be replaced except perhaps some global American postmodernist culture. (Jameson, 1986: 65)

É interessante notar que as "conversações recentes" são "entre intelectuais do terceiro mundo", podendo-se presumir que eles 
não fazem parte deste circuito de interlocução, embora vão emitir um juízo sobre ela. Por outro lado, a suposta descrição de um "retorno obsessivo" do nacional, daquela "velha coisa chamada nacionalismo", implica que este se encontrava em algum lugar discursivo recôndito ou anterior, de onde retorna à cena da fala. Tanto a referência a nós e ao "povo" (entre aspas, no texto) quanto as observações sobre atuações e características grupais parecem de algum modo remeter à questão das identidades coletivas, evocadas sob a rubrica de nacionalismo.

A ressalva de que este não é o modo como intelectuais norteamericanos têm discutido a "América" (também entre aspas, no texto), mas uma "velha coisa chamada 'nacionalismo', há muito tempo liquidada e com muita razão" chama a atenção principalmente pela descrição do nacionalismo como algo ultrapassado, e pela concordância do ensaísta com a liquidação que supõe estar descrevendo. Também é curioso que, embora declare o nacionalismo "liquidado" nos EUA, proponha que aquela velha coisa ainda "é fundamental no terceiro mundo (e também nas áreas mais vitais do segundo mundo)". Dai a sua pergunta retórica: - O nacionalismo é tão mau assim? Devemos encorajar o terceiro mundo a superálo tão rápido quanto possivel?

Acrescente-se que o ensaísta afirma não conseguir imaginar o que vai substituir aqueles nacionalismos, "exceto talvez alguma cultura pós-modernista americana global”, o que nos leva aos três adjetivos que qualificam o termo cultura, todos muito problemáticos.

Para começar, "pós-modernista" está longe de ser um adjetivo que possa mais consensualmente ser utilizado como designador de um certo periodo da contemporaneidade. Se para alguns encontramo-nos em plena pós-modernidade, vivendo sob condições de permanente e incurável incerteza - uma vida na presença de uma quantidade ilimitada de formas de vida, incapazes de provar suas aspirações a se fundamentarem em qualquer coisa mais sólida e amarrada do que suas próprias convenções historicamente molda- 
das (Bauman, 1993: 135) -, por outro lado, para outros, nem caberia falar em pós, se a modernidade ainda é um projeto incompleto. (Habermas, 1993).

Além disso, a justaposição dos adjetivos americana é global sintaticamente os coloca no mesmo plano, o que parece sugerir uma certa linha de raciocínio. semelhante à de João Ubaldo Ribeiro, que, respondendo à pergunta "Globalização ainda quer dizer alguma coisa?", declarou:

Esta pergunta toma como premissa a suposição de que eu sei o que quer dizer "globalização". Eu não sei. Ninguém sabe, aliás, porque não sou burro, leio bastante e não entendi nada dos que já tentaram definir a globalização. Pelo que percebo, no que acredito ser acompanhado pela maioria dos compatriotas, globalização são as empresas americanas mandando na gente. A cada dia, essa globalização avança e se solidifica e não vejo limites para sua expansão. Resta o consolo do brocardo latino quod abundat non nocet, que se traduz por "o que abunda não faz mal", afirmação quase sempre verdadeira, mas que, no caso da globalização, deve ser entendida como "quando a bunda não é nossa". (Ribeiro, 1999: 3)

É claro que sempre poderiamos questionar uma afirmativa tão crua quanto a do romancista brasileiro ("globalização são as empresas americanas mandando na gente"). Na opinião de Hans Ulrich Gumbrecht, por exemplo, talvez o McDonalds seja o único emblema de globalização realmente cem por cento norte-americano:

As coisas realmente importantes não são americanas. A pizza não é americana. A literatura mundial hoje em dia não é claramente americana. A moda, felizmente, não é americana. Os carros bons são alemães. E tanto a comida quanto a moda nos Estados Unidos melhoraram muito nas últimas décadas - graças à globalização!, que não exporta somente valores e 
conteúdos americanos. Então, é verdade que a globalização é uma relação negativa com identidades regionais, mas ao mesmo tempo talvez o que ela exporte sejam coisas da cultura ocidental. Hoje em dia você pode ter cachaças fenomenais na Alemanha Oriental, onde, por razões históricas, a cachaça é mais barata do que o conhaque francês. (Gumbrecht, 1999)

No que se refere à América Latina, George Yúdice, em artigo significativamente intitulado We are not the world, prefere falar em redes interculturais de disseminação e recepção, embora ressalte a assimetria destas redes. Para ele, não se pode falar do que a cultura dos Estados Unidos significa para os latino-americanos sem examinar seus meios de disseminação: filmes e programas de televisão, a imprensa popular, a cultura estética predominante, o jornalismo intelectual predominante (The New York Review of Books, The New York Times Book Review, Atlantic Monthly etc.) dos EUA e suas contraparțidas na América Latina. Yúdice acredita que a cultura acadêmica, para o melhor ou o pior, tem relativamente pouca influência na representação da cultura norte-americana na América Latina, de maneira que até agora o que é veiculado pela mídia predominante é, quase exclusivamente, o que chega ao público leitor e espectador latino-americano. No sentido inverso, o fluxo cultural da América Latina para os EUA, com poucas exceções, não seria mediado por instituições equivalentes de disseminação: decisões sobre quais filmes latino-americanos devem entrar em cartaz, que livros devem ser traduzidos, que movimentos sociais e políticos devem ser representados etc. seriam tomadas por iniciativas norteamericanas e pelo sistema universitário, que, em relação a isso, deteria considerável poder, especialmente em suas ligações com os aparatos formadores de políticas de Estado, mas também em esferas alternativas, como aquelas em que políticas de solidariedade e multiculturalismo interagem. (Yúdice, 1992: 204) 
JOBIN, José Luís. Atribuiçōes de identidade: o terceiro mundo, visto do primeiro, segundo...

Enfim, para terminar, convém não esquecer que o núcleo da argumentação de Jameson, neste artigo, também adota a hipótese de que todos os textos do terceiro mundo são alegorias nacionais. Nada melhor, neste ponto, do que recordar as próprias palavras do articulista, definindo o que as produções culturais do terceiro mundo parecem ter em comum e o que as distingue radicalmente de formas culturais análogas no primeiro mundo:

All third word texts are necessarily, I want to argue, allegorical, and in a very specific way: they are to be read as what I will call national allegories, even when, or perhaps I should say, particularly when their forms develop out of predominantly western machineries of representation, such as the novel. Let me try to state this distinction in a grossly oversimplified way: one of the determinants of capitalist culture, that is, the culture of the western realist and modernist novel, is a radical split between the private and the public, between the poetic and the political, between what we have come to think of as the domain of sexuality and the unconscious and that of the public world of classes, of the economic, and of secular political power: in other words, Freud versus Marx. Our numerous theoretical attempts to overcome this great split only reconfirm its existence and its shaping power over our individual and collective lives. We have been trained in a deep cultural conviction that the lived experience of our private existences is somehow incommensurable with the abstraction of economic science and political dynamics. Politics in our novels therefore is, according to Stendhal's canonical formulation, a "pistol shot in the middle of a concert."

I will argue that, although we may retain for convenience and for analysis such categories as the subjective and the public or political, the relations between them are wholly different in Third word culture. Third world texts, even those which are seemingly private and invested with a properly libidinal dynamic necessarily project a political dimension in the form of national allegory: the story of the private individual destiny is always an allegory of the embattled situation of the public Third word culture and society. Need I add that it is precisely this very 
different ratio of the political to the personal which makes such texts alien to us at first approach, and consequently, resistant to our conventional western habits of reading? (Jameson: 69)

O primeiro aspecto que ressaltaremos é: Jameson não faz observações sobre os textos, mas constrói uma teleologia da leitura, ao apregoar uma fórmula de interpretação para textos do terceiro mundo - eles devem ser lidos como alegorias nacionais, mesmo quando, ou especialmente quando suas formas se desenvolvem a partir de artefatos de representação predominantemente ocidentais, como o romance. Assim, a alegoria nacional não é algo que se possa presumir pertencer aos textos, mas alguma coisa que os leitores formulam, ao abordarem a literatura rotulada como "terceiromundista". Trata-se, portanto, de uma estratégia interpretativa, com aspiração a ter um âmbito de validade além de seu próprio formulador, embora não se detalhe mais explicitamente a que comunidade ou instituição poderia vincular-se esta estratégia. Também não se desenvolvem as implicações possiveis do sentido que se atribui àquela literatura, nem a vinculação ao lugar de produção deste sentido, com as respectivas questões daí derivadas. Por exemplo: - Se a literatura do terceiro mundo é aquela que pode ser lida como alegoria nacional, então a que eventualmente não puder ser lida como tal (penso em muitos textos de Clarice Lispector, por exemplo) deve ser excluida daquela categoria?

A aparente equalização entre o que Jameson chama de "cultura capitalista" e o romance realista e modernista (capitalist culture, that is, the culture of the western realist and modernist novel) já seria problemática, mas se torna mais problemática quando associada a uma divisão radical entre o privado e o público, entre sexualidadeinconsciente e economia-classes sociais-politica, entre Freud e Marx.

Como ressaltamos a questão da teleologia da leitura, terminaremos nosso percurso crítico observando que, como para o ensaísta norte-americano a alegoria nacional é uma fórmula interpretativa, então a aplicação desta fórmula vai determinar o. 
JOBIN, José Luís. Atribuições de identidade: o terceiro mundo, visto do primeiro, segundo...

que se vai encontrar. Ou seja, se Jameson afirma que os textos do terceiro mundo, mesmo aqueles mais aparentemente privados e investidos com uma dinâmica propriamente libidinal, necessariamente projetam uma dimensão politica na forma de alegoria nacional, isto é uma conseqüência e não uma causa da estratégia de leitura que apregoa, pois, se previamente está determinado que, mesmo as estórias dos destinos individuais necessariamente devem ser lidas como alegorias, então a prática de leitura que segue esta fórmula certamente vai encontrar no texto lido o que já era o princípio normativo da leitura. As observações que se fizerem serão de algum modo derivadas do próprio princípio normativo que se adotou para fazê-las: como a interpretação presume que o texto do terceiro mundo será uma alegoria nacional, então necessariamente o resultado da operação interpretativa será considerá-lo como... alegoria nacional. Há, portanto, uma certa tautologia nesta descoberta do que já se presumia existir, antes de começar a procurar.

Para terminar, devemos lembrar, a favor de Jameson, que ele próprio ressalva a precariedade de suas afirmações no ensaio, que ao ser republicado em livro ganha inclusive um novo título: World literature in the age of Multinational Capitalism.

\begin{abstract}
We will try to find out the key ideas through which Fredric Jameson, in his essay Third word Literature in the Era of Multinational Capital, builds up the frame of reference to ascribe a number of properties to what he calls "Third word Literature".
\end{abstract}

KEYWORDS: Identity of literature; third word. 


\section{Bibliografia}

AHMAD, A. (1987) "Jameson's Rethoric of Otherness and the 'National Allegory'”. In: Social Text, 17, p. 3-25.

ANDERSON, B. (1994) Imagined communities: reflections on the origin and spread of nationalism. London: Verso.

BAUMAN, Z. (1993) "The fall of the legislator". In: DOCHERTY, T. (Org.) Postmodernism - a reader. New York: Columbia University Press, p. 128-40.

BENJAMIN, C. et al. (1998) A opção brasileira. 5. ed. Porto Alegre: Contraponto.

HABERMAS, J. (1993) “Modernity - An Incomplete Project”. In: DOCHERTY, T. (Org.) Postmodernism - a reader. New York: Columbia University Press, p. 98-110.

JAMESON, F. (1986) "Third word Literature in the Era of Multinational Capital". In: Social Text, 15, p. 65-88.

. (1987) “A brief response". In: Social Text, 17, p. 26-7.

. (1987) "World literature in the age of Multinational Capitalism". In:

KOELBE and LOKKE (Ed.). The Current in Criticism. West Lafayette:

Purdue University Press, p. 139-58.

RIBEIRO, J. U. (1999) "João Ubaldo Ribeiro responde". In: Folha de S. Paulo, Caderno Mais!, 26.dez., p. 3.

SHOAT, E. (1992) "Notes on the 'post-colonial". In: Social Text, 31/32, p. 99-113.

YÜDICE, G. (1992) "We are not the world". In: Social Text, 31/32, p. 203-16. 